\title{
A REMARK ON THE DOUADY SEQUENCE FOR NON-PRIMARY HOPF MANIFOLDS *
}

\author{
XIANG-YU ZHOU ${ }^{\dagger}$ \\ Dedicated to Professor Yum-Tong Siu on his sixtieth birthday
}

1. Introduction. To determine cohomology groups of holomorphic vector bundles or more general coherent analytic sheaves on complex manifolds is very important in several complex variables and complex geometry. For example, Cartan-Serre's theorem B and Kodaira's vanishing theorem are fundamental respectively in the studies of two classes of complex manifolds: Stein manifolds and projective algebraic manifolds. Deformation and moduli space of complex structures are also closely related to the determination of the cohomology groups of the bundles according to Kodaira-Spencer's theory.

Some concrete calculations of cohomology groups on some well-known manifolds have been given. For example, cohomology groups of holomorphic line bundles (and more general holomorphic vector bundles with trivial pull back) on primary Hopf manifolds were calculated, where the Douady sequence (a short exact sequence of complexes, cf. $[1,4,10,11,14])$ plays a key role in the calculation.

The purpose of the present note is to generalize the Douady sequence to the case of non-primary Hopf manifolds, so that the cohomology groups of a holomorphic line bundles (and more general holomorphic vector bundles with trivial pull back) over arbitrary Hopf manifolds could be calculated. This could be used to obtain a criterion for a continuous complex vector bundle over any Hopf manifold admitting a holomorphic structure and to study the filtrability of the holomorphic vector bundles over any Hopf manifold and the moduli space of any Hopf manifold. (cf. [8-12,16,17])

\section{Some known results.}

A. By an $n$-dimentional Hopf manifold $X$, we shall mean a compact complex manifold of which the universal covering is $W=\mathbb{C}^{n}-0 . \quad X$ can be written as a quotient space $W / G$ with a group $G$ generated by some biholomorphic transformations of $W$ whose action on $W$ is properly discontinuous and free. When $n \geq 2$, it's known that $W$ is simply connected and $G$ is the fundamental group of $X$. When $G$ is the infinite cyclic group $\mathbb{Z}$, the Hopf manifold $X$ is called primary, otherwise it's called secondary or non-primary. Hopf manifold is the simplest complex manifold which is non-Kählerian.

Similar to Kodaira's statement for Hopf surface in [7], the fundamental group $G$ of $X$ has the following properties: $G$ contains a contraction $f$, and the infinite cyclic subgroup $\mathbb{Z}$ generated by $f$ has a finite index in $G$; the subgroup $\mathbb{Z}$ is located in the center of $G$ and then normal in $G$; the group $G / \mathbb{Z}$ is isomorphic to a finite subgroup $K$ of $G$.

*Received May 1, 2003; accepted for publication August 29, 2003. The research was supported by 973 Project Foundation of China and the Outstanding Youth Science Grant of NSFC (grant no. 19825105).

${ }^{\dagger}$ Department of Mathematics, Zhejiang University, Hangzhou, P.R. China; Institute of Mathematics, AMSS, Chinese Academy of Sciences, Beijing, P.R. China (xyzhou@mail2.math.ac.cn). 
Suppose that $A$ is a normal subgroup of the group $G, B$ is a subgroup of $G$, if there is an isomorphism $p$ between $G / A$ and $B$, then $G=A \cdot B$, a semi-direct product of $A$ and $B$. Actually, $\forall g \in G$, we have $p\left(g p(g)^{-1}\right)=e$, where $e$ is the identity of $G$ and $p(g) \in B$. This means that $g p(g)^{-1} \in A$. It's easy to see that $A \cap B=e$.

Therefore, the fundamental group $G$ of a Hopf manifold can be written as $G=$ $\mathbb{Z} \cdot K$ and $f \cdot k=k \cdot f$ for $\forall k \in K$.

B. A smooth complex vector bundle of rank $r$ over a real smooth manifold is called flat if it admits a flat structure, i.e., which is given by an open covering with local frame fields s.t. the transition functions are all constant matrices in $G L(r, \mathbb{C})$. It's well known that the bundle is flat is equivalent to that the bundle admits a flat connection in the sense that the curvature of the connection vanishes identically, and the bundle is defined by a representation of the fundamental group to $G L(r, \mathbb{C})$ (cf.[6]).

Using a similar argument of Kodaira's for Hopf surface in [7], we can get that any holomorphic line bundle on a Hopf manifold is flat.

Actually, given an arbitrary Hopf manifold $X$, there is a primary Hopf manifold $\tilde{X}$ and a holomorphic finite covering $p: \tilde{X} \rightarrow X$ with the finite group $K$ as the covering transformation group. Given any holomorphic line bundle on $X$, the pull back $p^{*}(L)$ on $\tilde{X}$ is flat. On the other hand, $K$ naturally acts on $p^{*}(L)$ and $L$ is isomorphic to $p^{*}(L) / K$. Based on a flat connection on $p^{*}(L)$, one can construct a $K$-invariant flat connection on $p^{*}(L)$ and then a flat connection on $p^{*}(L) / K$.

Assume $L \in H^{1}\left(X, \mathcal{O}^{*}\right)$ is a flat holomorphic line bundle on $X$, we can get the representation of the fundamental group $\pi_{1}(X) \cong \mathbb{Z} \cdot K$ of $X$ :

$$
\begin{aligned}
\rho: \pi_{1}(X) & \rightarrow G L(1, \mathbb{C}) \cong \mathbb{C}^{*} \\
f & \longmapsto c \\
k & \longmapsto \rho(k)
\end{aligned}
$$

where $f$ is the contraction which is the generator of $\mathbb{Z}$ and $k \in K$. The line bundle $L$ is the quotient of $W \times \mathbb{C}$, the trivial line bundle on the universal covering of $X$, by the operation of the representation of fundamental group in the following way:

$$
\begin{gathered}
g: W \times \mathbb{C} \rightarrow W \times \mathbb{C} \\
(z, v) \mapsto\left(g(z), \rho_{L}(g) v\right)
\end{gathered}
$$

where $g \in G$, i.e.,

$$
L=W \times \mathbb{C} / \pi_{1}(X)=W \times_{\rho} \mathbb{C}
$$

We define $\mathcal{O}(L)$ to be the locally free sheaf of germs of holomorphic local sections and $\Omega_{X}^{p}(L):=\Omega_{X}^{p} \otimes \mathcal{O}(L)$. We will present an observation to obtain a generalization of Douady exact sequence on any Hopf manifold, so that the groups $h^{q}\left(X, \Omega_{X}^{p}(L)\right):=\operatorname{dim} H^{q}\left(X, \Omega_{X}^{p}(L)\right)$ could be calculated for any Hopf manifold which is not necessarily primary.

C. A differential module $(\mathcal{K}, d)$ is a $R$-module $\mathcal{K}$ together with an endomorphism called differential $d: \mathcal{K} \rightarrow \mathcal{K}$ s.t. $d \circ d=0$. The homology group of $\mathcal{K}$ is by definition 
the quotient module $H(\mathcal{K})=Z(\mathcal{K}) / B(\mathcal{K})$, where $Z(\mathcal{K})=\operatorname{ker}(d), B(\mathcal{K})=\operatorname{Im}(d)$ are called respectively submodules of cycles and boundaries. A morphism of differential modules $\varphi:(\mathcal{K}, d) \rightarrow(\mathcal{L}, d)$ is a $R$-homomorphism $\varphi: \mathcal{K} \rightarrow \mathcal{L}$ s.t. $d \circ \varphi=\varphi \circ d$. It's easy to check that one can get an induced morphism on homology groups, denoted by $H(\varphi): H(\mathcal{K}) \rightarrow H(\mathcal{L})$.

It's known that one has the following so-called Snake Lemma. Given a short exact sequence of morphisms of differential modules $0 \rightarrow \mathcal{K} \stackrel{\varphi}{\rightarrow} \mathcal{L} \stackrel{\psi}{\rightarrow} \mathcal{M} \rightarrow 0$. Then there exists a connecting homomorphism $\partial: H(\mathcal{M}) \rightarrow H(\mathcal{K})$, and a homology exact sequence

$$
H(\mathcal{K}) \stackrel{H(\varphi)}{\longrightarrow} H(\mathcal{L}) \stackrel{H(\phi)}{\longrightarrow} H(\mathcal{M}) \stackrel{\partial}{\longrightarrow} H(\mathcal{K}) \stackrel{H(\varphi)}{\longrightarrow} H(\mathcal{L})
$$

The often used examples of differential modules are chain complex and cochain complex. A chain complex is a $Z$-graded differential module $K_{\bullet}=\bigoplus_{q \in \mathbb{Z}} K_{q}$ together with a differential $d$ of degree -1, i.e., $d=\bigoplus d_{q}$ with $d_{q}: K_{q} \rightarrow K_{q-1}$ and $d_{q-1} \circ d_{q}=$ 0 . Similarly, a cochain complex is a $Z$-graded differential module $K^{\bullet}=\bigoplus_{q \in \mathbb{Z}} K^{q}$ together with a differential $d$ of degree 1, i.e., $d=\bigoplus d^{q}$ with $d^{q}: K^{q} \rightarrow K^{q+1}$ and $d^{q+1} \circ d^{q}=0$. Morphisms of complexes are assumed to be of degree 0 , i.e., of the form $\varphi=\bigoplus \varphi^{q}$ with $\varphi^{q}: K^{q} \rightarrow L^{q}$.

By the Snake Lemma, any short exact sequence

$$
0 \rightarrow K^{\bullet} \stackrel{\varphi}{\rightarrow} L^{\bullet} \stackrel{\psi}{\rightarrow} M^{\bullet} \rightarrow 0
$$

gives rise to a corresponding long exact sequence of cohomology groups

$$
\cdots \rightarrow H^{q}\left(K^{\bullet}\right) \stackrel{H^{q}(\varphi)}{\longrightarrow} H^{q}\left(L^{\bullet}\right) \stackrel{H^{q}(\phi)}{\longrightarrow} H^{q}\left(M^{\bullet}\right) \stackrel{\partial^{q}}{\longrightarrow} H^{q+1}\left(K^{\bullet}\right) \rightarrow \cdots
$$

3. Main result. To take an open covering $\mathcal{A}=\left\{U_{i}\right\}$ of $X$ such that all $U_{i}$ are contractible Stein subsets of $X$ and $\tilde{U}_{i}:=\pi^{-1}\left(U_{i}\right)$ is a disjoint union of open Stein subsets $\left\{U_{i j k}^{\prime}\right\}$ of $\mathrm{W}$, each of them isomorphic to $U_{i}$. We rewrite

$$
\begin{gathered}
\tilde{U}_{i}:=\bigcup_{j \in \mathbb{Z}} f^{j}\left(\bigcup_{k \in K} k\left(U_{i 00}^{\prime}\right)\right), U_{i 00}^{\prime} \cong U_{i}, U_{i j k}^{\prime}:=f^{j} k U_{i 00}^{\prime}, \\
\tilde{U}_{i}=\bigcup_{j \in \mathbb{Z}, k \in K} U_{i j k}^{\prime}
\end{gathered}
$$

$\tilde{\mathcal{A}}:=\left\{\tilde{U}_{i}\right\}$ is a covering of $W$, where $K$ is given in 2.A. If $\varphi \in \Gamma\left(U_{i}, \Omega_{X}^{p}(L)\right.$, then $\tilde{\varphi}:=$ $\pi^{*}(\varphi)$ contained in $\Gamma\left(\tilde{U}_{i}, \pi^{*}\left(\Omega_{X}^{p}(L)\right)\right)$. Since $\pi^{*}\left(\Omega_{X}^{p}(L)\right)=\pi^{*}\left(\Omega_{X}^{p}\right) \otimes \pi^{*}(L), \pi^{*}(L)=$ $\mathcal{O}_{W}$, and $\pi^{*}\left(\Omega_{X}^{p}\right)=\Omega_{W}^{p}$, we have $\tilde{\varphi} \in \Gamma\left(\tilde{U}_{i}, \Omega_{W}^{p}\right)$, thus we can establish a sequence of Čech complexes:

$$
0 \rightarrow C^{\bullet}\left(\mathcal{A}, \Omega_{X}^{p}(L)\right) \stackrel{\pi^{*}}{\rightarrow} S^{\bullet} \stackrel{c I d-f^{*}}{\longrightarrow} S^{\bullet} \rightarrow 0
$$

where $S^{\bullet}:=C^{\bullet}\left(\tilde{\mathcal{A}}, \Omega_{W}^{p}\right)^{K}=\operatorname{ker}\left\{\rho(k) I d-k^{*}: C^{\bullet}\left(\tilde{\mathcal{A}}, \Omega_{W}^{p}\right) \rightarrow C^{\bullet}\left(\tilde{\mathcal{A}}, \Omega_{W}^{p}\right), \forall k \in K\right\} \subset$ $C^{\bullet}\left(\tilde{\mathcal{A}}, \Omega_{W}^{p}\right)$.

Our main observation is the following:

Theorem. The short sequence (1) of $\check{C}$ ech complexes is exact. 
Proof. 1. We show first the surjectivity of map $c I d-f^{*}$.

Without loss of generality, let $\gamma$ be an element of $\Gamma\left(\tilde{U}_{i_{0}} \cap \cdots \cap \tilde{U}_{i_{q}}, \Omega_{W}^{p}\right)$ satisfying $K$ invariant i.e., $k^{*} \gamma=\rho(k) \gamma$, for $\forall k \in K$. It is clear that

$$
\tilde{U}_{i_{0}} \cap \cdots \cap \tilde{U}_{i_{q}}=\bigcup_{j \in \mathbb{Z}} f^{j}\left(\bigcup_{k \in K} k\left(U_{i_{0} 00}^{\prime} \cap \cdots \cap U_{i_{q} 00}^{\prime}\right)\right)
$$

We put $\gamma=\gamma_{1}-\gamma_{2}$ where

$$
\gamma_{1}= \begin{cases}\gamma, & z \in \bigcup_{j \geq 0} f^{j}\left(\bigcup_{k \in K} k\left(U_{i_{0} 00}^{\prime} \cap \cdots \cap U_{i_{q} 00}^{\prime}\right)\right. \\ 0 & z \in \bigcup_{j<0} f^{j}\left(\bigcup_{k \in K} k\left(U_{i_{0} 00}^{\prime} \cap \cdots \cap U_{i_{q} 00}^{\prime}\right)\right.\end{cases}
$$

and

$$
\gamma_{2}= \begin{cases}0, & z \in \bigcup_{j \geq 0} f^{j}\left(\bigcup_{k \in K} k\left(U_{i_{0} 00}^{\prime} \cap \cdots \cap U_{i_{q} 00}^{\prime}\right)\right. \\ -\gamma, & z \in \bigcup_{j<0} f^{j}\left(\bigcup_{k \in K} k\left(U_{i_{0} 00}^{\prime} \cap \cdots \cap U_{i_{q} 00}^{\prime}\right)\right.\end{cases}
$$

set

$$
\beta:=\sum_{j \geq 0} c^{-j-1}\left(f^{*}\right)^{j}\left(\gamma_{1}\right)+\sum_{j<0} c^{-j-1}\left(f^{*}\right)^{j}\left(\gamma_{2}\right)
$$

It is well defined since the sums are locally finite.

0 .

It is clear that $\gamma_{1}, \gamma_{2}$ is $K$ invariant, i.e., $\left(\rho(k) I d-k^{*}\right) \gamma_{1}=0$ and $\left(\rho(k) I d-k^{*}\right) \gamma_{2}=$

Since $\mathbb{Z}$ belongs to the center of $G$, i.e. $f \cdot k=k \cdot f$, so $\beta$ is also $K$ invariant.

Therefore

$$
\begin{aligned}
f^{*} \beta & =\sum_{j \geq 0} f^{*}\left(c^{-j-1}\left(f^{*}\right)^{j}\left(\gamma_{1}\right)\right)+\sum_{j<0} f^{*}\left(c^{-j-1}\left(f^{*}\right)^{j}\left(\gamma_{2}\right)\right) \\
& =\sum_{j \geq 0} c^{-j-1}\left(f^{*}\right)^{j+1}\left(\gamma_{1}\right)+\sum_{j<0} c^{-j-1}\left(f^{*}\right)^{j+1}\left(\gamma_{2}\right) \\
& =c\left[\sum_{j \geq 0} c^{-(j+1)-1}\left(f^{*}\right)^{j+1}\left(\gamma_{1}\right)+\sum_{j<0} c^{-(j+1)-1}\left(f^{*}\right)^{j+1}\left(\gamma_{2}\right)\right] \\
& =c\left[\sum_{j \geq 1} c^{-j-1}\left(f^{*}\right)^{j}\left(\gamma_{1}\right)+\sum_{j<1} c^{-j-1}\left(f^{*}\right)^{j}\left(\gamma_{2}\right)\right]
\end{aligned}
$$

By definition:

$$
c \beta=c\left[\sum_{j \geq 0} c^{-j-1}\left(f^{*}\right)^{j}\left(\gamma_{1}\right)+\sum_{j<0} c^{-j-1}\left(f^{*}\right)^{j}\left(\gamma_{2}\right)\right]
$$

From the above we get

$$
\left(c I d-f^{*}\right) \beta=c \beta-f^{*} \beta=\gamma_{1}-\gamma_{2}
$$

So the proof of surjectivity is completed.

2. To prove the injectivity of $\pi^{*}$ and $\operatorname{Im} \pi^{*}=k e r\left(c I d-f^{*}\right)$. 
Let $\gamma \in \Gamma\left(U_{i_{0}} \cap \cdots \cap U_{i_{q}}, \Omega_{X}^{p}(L)\right)$, from the representation of $L$, we know

$$
\pi^{*} \gamma \in \Gamma\left(\tilde{U}_{i_{0}} \cap \cdots \cap \tilde{U}_{i_{q}}, \Omega_{W}^{p}\right)
$$

which can be presented by $\left\{\cdots, \tilde{\gamma}_{-k}, \cdots, \tilde{\gamma}_{-1}, \tilde{\gamma}_{0}, \tilde{\gamma}_{1}, \cdots, \tilde{\gamma}_{k}, \cdots\right\}$, where

$$
\tilde{\gamma}_{k} \in \Gamma\left(f^{k}\left(\bigcup_{k \in K} k\left(U_{i_{0} 00}^{\prime} \cap \cdots \cap U_{i_{q} 00}^{\prime}\right)\right), \Omega_{W}^{p}\right)
$$

and $\tilde{\gamma}_{k}\left(f^{k}(z)\right)=c^{k} \tilde{\gamma}_{0}(z)$, for any $z \in \bigcup_{k \in K} k\left(U_{i_{0} 00}^{\prime} \cap \cdots \cap U_{i_{q} 00}^{\prime}\right)$

We derive from the above the injectivity of the map $\pi^{*}$ and

$$
f^{*} \circ\left(\pi^{*} \circ \gamma\right)(z)=\pi^{*} \circ \gamma(f(z))=c \pi^{*} \circ \gamma(z)
$$

thus

$$
\left(c I d-f^{*}\right) \circ \pi^{*} \circ \gamma=0
$$

Similarly, we can get

$$
k^{*}\left(\pi^{*} \circ \gamma\right)=\rho(k) \pi^{*} \circ \gamma
$$

Therefore, we have

$$
\operatorname{Im} \pi^{*} \subseteq \operatorname{ker}\left(c I d-f^{*}\right)
$$

Take any $\tilde{\gamma} \in \Gamma\left(\tilde{U}_{i_{0}} \cap \cdots \cap \tilde{U}_{i_{q}}, \Omega_{W}^{p}\right)$ s.t $f^{*} \tilde{\gamma}=c \tilde{\gamma}$ and $k^{*} \tilde{\gamma}=\rho(k) \tilde{\gamma}$, and $G=\mathbb{Z} \cdot K$, so there exists $\gamma \in \Gamma\left(U_{i_{0}} \cap \cdots \cap U_{i_{q}}, \Omega_{X}^{p}(L)\right)$ s.t. $\pi^{*} \gamma=\tilde{\gamma}$, therefore

$$
\operatorname{Im} \pi^{*} \supseteq \operatorname{ker}\left(c I d-f^{*}\right)
$$

The proof of the theorem is finished.

REMARK. When $K$ is trivial, we can get as a special case the usual Douady sequence as used in $[1,4,10,11,15]$. When $G$ is Abelian, the Douady sequence was obtained in $[16,17]$.

Corollary. By 2.C and Leray's theorem, we can get the long exact sequence of cohomology

$$
\begin{gathered}
0 \rightarrow H^{0}\left(X, \Omega_{X}^{p}(L)\right) \rightarrow H^{0}\left(\tilde{\mathcal{A}}, S^{\bullet}\right) \stackrel{P_{0}}{\longrightarrow} H^{0}\left(\tilde{\mathcal{A}}, S^{\bullet}\right) \rightarrow H^{1}\left(X, \Omega_{X}^{p}(L)\right) \rightarrow \\
\rightarrow H^{1}\left(\tilde{\mathcal{A}}, S^{\bullet}\right) \stackrel{P_{1}}{\longrightarrow} H^{1}\left(\tilde{\mathcal{A}}, S^{\bullet}\right) \rightarrow H^{2}\left(X, \Omega_{X}^{p}(L)\right) \rightarrow \\
\vdots \\
\rightarrow H^{q-1}\left(\tilde{\mathcal{A}}, S^{\bullet}\right) \stackrel{P_{q-1}}{\longrightarrow} H^{q-1}\left(\tilde{\mathcal{A}}, S^{\bullet}\right) \rightarrow H^{q}\left(X, \Omega_{X}^{p}(L)\right) \rightarrow \cdots
\end{gathered}
$$

where $P:=c I d-f^{*}, P_{q}:=H^{q}(P)$. 
REMARK. The group $H^{q}\left(\tilde{\mathcal{A}}, S^{\bullet}\right)$ is related to the cohomology group of the induced line bundle of $L$ over $W / K$ (where $K$ is a finite group), which is not hard to calculate. In this way, one could compute the cohomology groups of holomorphic line bundles over any Hopf manifold. And then one can use the calculations to determine when the complex vector bundles over any Hopf manifolds admits holomorphic structures and the holomorphic vector bundles over any Hopf manifolds are filtrable (for details we refer to $[9,16,17])$. The main result could also be extended to the case of holomorphic vector bundles with trivial pullback.

\section{REFERENCES}

[1] C. BorceA, Some remarks on deformations of Hopf manifolds, Rev. Roum. Math. Pures et Appl., 26:10 (1981), pp. 1287-1294.

[2] K. Dabrowski, Moduli spaces for Hopf surfaces, Math Ann., 259 (1982), pp. 201-225.

[3] J.P. Demailly, Complex analytic and differential geometry, Unpublished, 1997.

[4] A. DouAdy, Seminare H.Cartan, Expose 3, 1960/1961.

[5] M. Kato, Topology of Hopf surfaces, J. Math. Soc. Japan, 27:2 (1975).

[6] S. Kobayashi, Differential geometry of complex vector bundles, Iwanami Shoten, publishers and Princeton University Press, 1987.

[7] K. Kodaira, On the structure of compact complex analytic surfaces, Am. J. .Math., 86 (1964), pp. 751-798; 88 (1966), pp. 682-721; 90 (1968), pp. 55-83.

[8] Q.Z. Li And J.H. Zhang, The Moduli space of Hopf surfaces, Science in China (Series A), $38: 9$ (1995), pp. 1049-1059.

[9] Weimin LiU And Xiang-Yu Zhou, Holomorphic vector bundles on Hopf manifolds with Abelian fundamental group, to appear in "Acta Math. Sinica".

[10] D. MALL, The cohomology of line bundles on Hopf Manifolds, Osaka J. Math, 28 (1991), pp. 999-1015.

[11] D. MALL, Contractions, Fredholm operators and the cohomology of vector bundles on Hopf manifolds, Arch. Math., 66 (1996), pp. 71-76.

[12] D. MALL, On holomorphic vector bundle on Hopf Manifolds with pullback on the universal covering, Math.Ann, 294 (1992), pp. 791-740.

[13] T. Matumoto and N. Nakagawa, Explicit description of Hopf surfaces and their automorphism groups, Osaka J. Math., 37 (2000), pp. 417-424.

[14] T. том Dieck, Transformation groups, Walter de Gruyter, Berlin-New York, 1987.

[15] J. WeHLER, Versal deformation of Hopf surfaces, J. Reine. angew. Math., 328 (1981), pp. $22-32$.

[16] Xiang-Yu Zhou and Weimin Liu, Line bundles on non-primary Hopf manifolds, to appear in "Science in China".

[17] Xiang-Yu Zhou And Weimin Liu, Some results of holomorphic vector bundles on non-primary Hopf manifolds, Advances in Math. (China), 32:2 (2003), pp. 246-248. 